\title{
Linear polarization of gluons and Higgs plus jet production at the LHC
}

\section{Cristian PISANO*}

Department of Physics, University of Antwerp, Groenenborgerlaan 171, 2020 Antwerp, Belgium

E-mail: cristian.pisano@uantwerp.be

\begin{abstract}
We show that the production of a Higgs boson in association with a jet in proton-proton collisions is sensitive to the linear polarization of gluons inside unpolarized protons. We present the analytical expressions, at leading order in perturbative QCD, for various transverse momentum dependent observables, which can be measured at the LHC. Since there are no experimental constraints on the linearly polarized gluon distribution, its effects are studied by adopting two different models. In particular, we find that the $\cos 2 \phi$ azimuthal asymmetry is a very promising observable because it could give access to the sign of this new distribution.
\end{abstract}

XXIII International Workshop on Deep-Inelastic Scattering 27 April - May 12015

Dallas, Texas

* Speaker. 


\section{Introduction}

Gluons can be linearly polarized, even inside an unpolarized hadron, if one takes into account their transverse momentum with respect to the hadron momentum [1]. The amount of polarization, corresponding to an interference between +1 and -1 gluon helicity states, is so far unknown. However, if sufficiently large it could provide, for instance, a valuable new tool to analyze the couplings of the Higgs boson to the standard model particles into which it can decay [2]. The effects of gluon polarization can be studied in a convenient way adopting the formalism of transverse momentum dependent (TMD) parton distribution functions (TMDs, for short). Within this framework, inclusive Higgs production has been studied in Refs. [2,3] and, including the effects of TMD evolution, in Refs. [4, 5]. The impact of gluon polarization is expected to be small, most likely a few percent, at the energy scale of the Higgs mass $M_{H}$. Moreover, these effects are largest when the transverse momentum of the Higgs boson is small, i.e. a few $\mathrm{GeV}$, where the cross section is difficult to measure.

Alternatively, in the following, we propose the study of Higgs production in association with a jet [6]. When it comes to probe the linear polarization of gluons, this process offers some additional features compared to inclusive Higgs production. First of all, one can study the TMD evolution by tuning the hard scale, identified for example with the invariant mass of the Higgs-jet pair. This is not possible in Higgs production, when the hard scale is fixed to be $M_{H}$. Furthermore, it is possible to define angular modulations, which have the advantage of singling out specific contributions. Finally, we note that the effects of gluon polarization show up in the transverse momentum distribution of the Higgs-jet pair, where the transverse momentum of the pair can be as small as a few $\mathrm{GeV}$, but the single transverse momenta of the Higgs boson and the jet might be much larger.

\section{Theoretical framework}

We consider the process $p\left(P_{A}\right)+p\left(P_{B}\right) \rightarrow H\left(K_{H}\right)+$ jet $\left(K_{\mathrm{j}}\right)+X$, where the four-momenta of the particles are given within brackets, and the Higgs boson and the jet are almost back to back in the plane perpendicular to the direction of the incoming protons. At leading order in perturbative QCD, the partonic subprocesses that contribute are $g g \rightarrow H g, g q \rightarrow H q$ and $q \bar{q} \rightarrow H g$. We perform a Sudakov decomposition of the initial hadronic momenta $P_{A}$ and $P_{B}$ in terms of the lightlike vectors $n_{+}$and $n_{-}$, with $n_{+} \cdot n_{-}=1$ :

$$
P_{A}^{\mu}=P_{A}^{+} n_{+}^{\mu}+\frac{M_{p}^{2}}{2 P_{A}^{+}} n_{-}^{\mu}, \quad P_{B}^{\mu}=\frac{M_{p}^{2}}{2 P_{B}^{-}} n_{+}^{\mu}+P_{B}^{-} n_{-}^{\mu},
$$

where $M_{p}$ is the proton mass. Similarly, one can express the momenta of the two incoming partons, $p_{a}$ and $p_{b}$, as follows

$$
p_{a}^{\mu}=x_{a} P_{A}^{+} n_{+}^{\mu}+\frac{p_{a}^{2}+\boldsymbol{p}_{a T}^{2}}{2 x_{a} P_{A}^{+}} n_{-}^{\mu}+p_{a T}^{\mu}, \quad p_{b}^{\mu}=\frac{p_{b}^{2}+\boldsymbol{p}_{b T}^{2}}{2 x_{b} P_{B}^{-}} n_{+}^{\mu}+x_{b} P_{B}^{-} n_{-}^{\mu}+p_{b T}^{\mu},
$$

with $x_{a}, x_{b}$ being the partonic light cone momentum fractions and $p_{a T}, p_{b T}$ the intrinsic transverse momenta. Moreover, we define the difference and sum of the final transverse momenta, $\boldsymbol{K}_{\perp}=$ $\left(\boldsymbol{K}_{H \perp}-\boldsymbol{K}_{\mathrm{j} \perp}\right) / 2$ and $\boldsymbol{q}_{T}=\boldsymbol{K}_{H \perp}+\boldsymbol{K}_{\mathrm{j} \perp}$, with $\left|\boldsymbol{q}_{T}\right| \ll\left|\boldsymbol{K}_{\perp}\right|$ because the Higgs and jet transverse momenta are almost opposite. The azimuthal angle between $\boldsymbol{K}_{\perp}$ and $\boldsymbol{q}_{T}$ is denoted by $\phi \equiv \phi_{\perp}-\phi_{T}$. 
Restricting ourselves to the channel $g g \rightarrow H g$, which is the dominant one at the LHC energies, and assuming TMD factorization, the cross section for the process $p p \rightarrow H$ jet $X$ is given by

$$
\begin{aligned}
\mathrm{d} \sigma=\frac{1}{2 s} \frac{d^{3} \boldsymbol{K}_{H}}{(2 \pi)^{3} 2 E_{H}} & \frac{d^{3} \boldsymbol{K}_{\mathrm{j}}}{(2 \pi)^{3} 2 E_{\mathrm{j}}} \int \mathrm{d} x_{a} \mathrm{~d} x_{b} \mathrm{~d}^{2} \boldsymbol{p}_{a T} \mathrm{~d}^{2} \boldsymbol{p}_{b T}(2 \pi)^{4} \delta^{4}\left(p_{a}+p_{b}-K_{H}-K_{\mathrm{j}}\right) \\
& \times \operatorname{Tr}\left\{\Phi_{g}^{[U]}\left(x_{a}, \boldsymbol{p}_{a T}\right) \Phi_{g}^{[U]}\left(x_{b}, \boldsymbol{p}_{b T}\right)\left|\mathscr{M}^{g g \rightarrow H g}\left(p_{a}, p_{b} ; K_{H}, K_{\mathrm{j}}\right)\right|^{2}\right\},
\end{aligned}
$$

where $s=\left(P_{A}+P_{B}\right)^{2}$, the trace is taken over the Lorentz indices and $\mathscr{M}^{g g \rightarrow H g}$ is the amplitude for the partonic subprocess. The correlators $\Phi_{g}^{[U]}$, describing the proton $\rightarrow$ gluon transitions, are defined in terms of matrix elements of the gluon field strengths $F^{\mu v}(0)$ and $F^{\mu v}(\xi)$ on the light front $(\mathrm{LF}) \xi \cdot n \equiv 0[1]$,

$$
\begin{aligned}
\Phi_{g}^{[U] \mu v}\left(x, \boldsymbol{p}_{T}\right) & \left.=\frac{n_{\rho} n_{\sigma}}{(p \cdot n)^{2}} \int \frac{\mathrm{d}(\xi \cdot P) \mathrm{d}^{2} \xi_{T}}{(2 \pi)^{3}} e^{i p \cdot \xi}\left\langle P\left|\operatorname{Tr}\left[F^{\mu \rho}(0) U_{[0, \xi]} F^{v \sigma}(\xi) U_{[\xi, 0]}^{\prime}\right]\right| P\right\rangle\right\rfloor_{\mathrm{LF}} \\
& =-\frac{1}{2 x}\left\{g_{T}^{\mu v} f_{1}^{g}\left(x, \boldsymbol{p}_{T}^{2}\right)-\left(\frac{p_{T}^{\mu} p_{T}^{v}}{M_{p}^{2}}+g_{T}^{\mu v} \frac{\boldsymbol{p}_{T}^{2}}{2 M_{p}^{2}}\right) h_{1}^{\perp g}\left(x, \boldsymbol{p}_{T}^{2}\right)\right\},
\end{aligned}
$$

with $n \equiv n_{-}$for gluon $a$ with momentum $p_{a}$ and $n \equiv n_{+}$for gluon $b$ with momentum $p_{b}$. The process dependent gauge links $U_{[0, \xi]}$ and $U_{[\xi, 0]}^{\prime}$ are needed to render the correlator gauge invariant, while the transverse tensor $g_{T}^{\mu \nu}$ is defined as $g_{T}^{\mu \nu}=g^{\mu \nu}-n_{+}^{\mu} n_{-}^{\nu}-n_{-}^{\mu} n_{+}^{v}$. The unpolarized and linearly polarized gluon TMD distributions are denoted respectively by $f_{1}^{g}\left(x, \boldsymbol{p}_{T}^{2}\right)$ and $h_{1}^{\perp g}\left(x, \boldsymbol{p}_{T}^{2}\right)$, and satisfy the model-independent relation [1]

$$
\frac{\boldsymbol{p}_{T}^{2}}{2 M_{p}^{2}}\left|h_{1}^{\perp g}\left(x, \boldsymbol{p}_{T}^{2}\right)\right| \leq f_{1}^{g}\left(x, \boldsymbol{p}_{T}^{2}\right) .
$$

Since $h_{1}^{\perp g}$ is $T$-even, it can be nonzero in absence of initial and/or final state interactions. However, such interactions can render it process dependent, like all other TMDs, or even lead to the breaking of factorization. Here we will not consider such effects. We just note that there are at present no indications that factorization may be broken in $p p \rightarrow H$ jet $X$ because of color entanglement as in $p p \rightarrow$ jetjet $X[8]$.

From Eqs. (2.3), (2.4) and the explicit expression for the amplitude $\mathscr{M}^{g g \rightarrow H g}$, one can calculate the normalized cross section for the process $p p \rightarrow H$ jet $X$, defined as

$$
\frac{\mathrm{d} \sigma}{\sigma} \equiv \frac{\mathrm{d} \sigma}{\int_{0}^{q_{T \max }^{2}} \mathrm{~d} \boldsymbol{q}_{T}^{2} \int_{0}^{2 \pi} \mathrm{d} \phi \mathrm{d} \sigma}, \quad \text { with } \quad \mathrm{d} \sigma \equiv \frac{\mathrm{d} \sigma}{\mathrm{d} y_{H} \mathrm{~d} y_{\mathrm{j}} \mathrm{d}^{2} \boldsymbol{K}_{\perp} \mathrm{d}^{2} \boldsymbol{q}_{T}},
$$

where $y_{H}$ and $y_{j}$ are, respectively, the rapidities of the produced Higgs boson and jet along the direction of the incoming protons. By neglecting terms suppressed by powers of $\left|\boldsymbol{q}_{T}\right| / M_{\perp}$, with $M_{\perp}=\sqrt{M_{H}^{2}+K_{H \perp}^{2}}$, the final result in the laboratory frame takes the form

$$
\frac{\mathrm{d} \sigma}{\sigma}=\frac{1}{2 \pi} \sigma_{0}\left(\boldsymbol{q}_{T}^{2}\right)\left[1+R_{0}\left(\boldsymbol{q}_{T}^{2}\right)+R_{2}\left(\boldsymbol{q}_{T}^{2}\right) \cos 2 \phi+R_{4}\left(\boldsymbol{q}_{T}^{2}\right) \cos 4 \phi\right]
$$

where

$$
\sigma_{0}\left(\boldsymbol{q}_{T}^{2}\right) \equiv \frac{\mathscr{C}\left[f_{1}^{g} f_{1}^{g}\right]}{\int_{0}^{q_{T \max }^{2}} \mathrm{~d} \boldsymbol{q}_{T}^{2} \mathscr{C}\left[f_{1}^{g} f_{1}^{g}\right]},
$$


and we have introduced the convolution of TMDs

$$
\mathscr{C}[w f g] \equiv \int d^{2} \boldsymbol{p}_{a T} \int d^{2} \boldsymbol{p}_{b T} \delta^{2}\left(\boldsymbol{p}_{a T}+\boldsymbol{p}_{b T}-\boldsymbol{q}_{T}\right) w\left(\boldsymbol{p}_{a T}, \boldsymbol{p}_{b T}\right) f\left(x_{a}, \boldsymbol{p}_{a T}^{2}\right) g\left(x_{b}, \boldsymbol{p}_{b T}^{2}\right),
$$

with, up to corrections of order $\mathscr{O}(1 / s), x_{a / b}=\left(M_{\perp} e^{ \pm y_{H}}+\left|\boldsymbol{K}_{\mathrm{j} \perp}\right| e^{ \pm y_{\mathrm{j}}}\right) / \sqrt{s}$. The different terms $R_{0}, R_{2}$ and $R_{4}$ are functions of the Mandelstam variables $\hat{s}, \hat{t}, \hat{u}$ for the subprocess $g g \rightarrow H g$ and contain convolutions of the gluon TMDs. They read:

$$
\begin{aligned}
& R_{0}\left(\boldsymbol{q}_{T}^{2}\right)=\frac{M_{H}^{4} \hat{s}^{2}}{M_{H}^{8}+\hat{s}^{4}+\hat{t}^{4}+\hat{u}^{4}} \frac{\mathscr{C}\left[w_{0}^{h h} h_{1}^{\perp g} h_{1}^{\perp g}\right]}{\mathscr{C}\left[f_{1}^{g} f_{1}^{g}\right]}, \\
& R_{2}\left(\boldsymbol{q}_{T}^{2}\right)=\frac{\hat{t}^{2}(\hat{t}+\hat{u})^{2}-2 M_{H}^{2} \hat{u}^{2}(\hat{t}+\hat{u})+M_{H}^{4}\left(\hat{t}^{2}+\hat{u}^{2}\right)}{M_{H}^{8}+\hat{s}^{4}+\hat{t}^{4}+\hat{u}^{4}} \frac{\mathscr{C}\left[w_{2}^{f h} f_{1}^{g} h_{1}^{\perp g}\right]}{\mathscr{C}\left[f_{1}^{g} f_{1}^{g}\right]}+\left(x_{a} \leftrightarrow x_{b}, \hat{t} \leftrightarrow \hat{u}\right), \\
& R_{4}\left(\boldsymbol{q}_{T}^{2}\right)=\frac{\hat{t}^{2} \hat{u}^{2}}{M_{H}^{8}+\hat{s}^{4}+\hat{t}^{4}+\hat{u}^{4}} \frac{\mathscr{C}\left[w_{4}^{h h} h_{1}^{\perp g} h_{1}^{\perp g}\right]}{\mathscr{C}\left[f_{1}^{g} f_{1}^{g}\right]},
\end{aligned}
$$

with the transverse weights given by

$$
\begin{aligned}
& w_{0}^{h h}=\frac{1}{M_{p}^{4}}\left[\left(\boldsymbol{p}_{a T} \cdot \boldsymbol{p}_{b T}\right)^{2}-\frac{1}{2} \boldsymbol{p}_{a T}^{2} \boldsymbol{p}_{b T}^{2}\right], \\
& w_{2}^{f h}=\frac{1}{M_{p}^{2}}\left[2 \frac{\left(\boldsymbol{q}_{T} \cdot \boldsymbol{p}_{b T}\right)^{2}}{\boldsymbol{q}_{T}^{2}}-\boldsymbol{p}_{b T}^{2}\right], \quad w_{2}^{h f}=\frac{1}{M_{p}^{2}}\left[2 \frac{\left(\boldsymbol{q}_{T} \cdot \boldsymbol{p}_{a T}\right)^{2}}{\boldsymbol{q}_{T}^{2}}-\boldsymbol{p}_{a T}^{2}\right], \\
& w_{4}^{h h}=\frac{1}{2 M_{p}^{4}}\left\{2\left[2 \frac{\left(\boldsymbol{q}_{T} \cdot \boldsymbol{p}_{a T}\right)\left(\boldsymbol{q}_{T} \cdot \boldsymbol{p}_{b T}\right)}{\boldsymbol{q}_{T}^{2}}-\boldsymbol{p}_{a T} \cdot \boldsymbol{p}_{b T}\right]^{2}-\boldsymbol{p}_{a T}^{2} \boldsymbol{p}_{b T}^{2}\right\} .
\end{aligned}
$$

In order to single out the different terms $1+R_{0}, R_{2}, R_{4}$ in Eq. (2.7), we define the observables [7]

$$
\langle\cos n \phi\rangle_{q_{T}} \equiv \frac{\int_{0}^{2 \pi} \mathrm{d} \phi \cos n \phi \mathrm{d} \sigma}{\sigma}, \quad n=0,2,4,
$$

such that their integrals over $\boldsymbol{q}_{T}^{2}$ give the average values of $\cos n \phi$. Namely,

$$
\langle\cos n \phi\rangle \equiv \frac{\int_{0}^{q_{T \max }^{2}} \mathrm{~d} \boldsymbol{q}_{T}^{2} \int_{0}^{2 \pi} \mathrm{d} \phi \cos n \phi \mathrm{d} \sigma}{\sigma}=\int_{0}^{q_{T \max }^{2}} \mathrm{~d} \boldsymbol{q}_{T}^{2}\langle\cos n \phi\rangle_{q_{T}}, \quad n=0,2,4
$$

The choice of $q_{T \max }$ must be consistent with the requirement of TMD factorization that $q_{T} \ll Q$, with $Q$ being the hard scale of the process. Here we take $q_{T \max }=M_{H} / 2$ [6]. It can be shown that

$$
\begin{aligned}
\frac{1}{\sigma} \frac{\mathrm{d} \sigma}{\mathrm{d} \boldsymbol{q}_{T}^{2}} & \equiv\langle 1\rangle_{q_{T}}=\sigma_{0}\left(\boldsymbol{q}_{T}^{2}\right)\left[1+R_{0}\left(\boldsymbol{q}_{T}^{2}\right)\right], \\
\langle\cos 2 \phi\rangle_{q_{T}} & =\frac{1}{2} \sigma_{0}\left(\boldsymbol{q}_{T}^{2}\right) R_{2}\left(\boldsymbol{q}_{T}^{2}\right), \\
\langle\cos 4 \phi\rangle_{q_{T}} & =\frac{1}{2} \sigma_{0}\left(\boldsymbol{q}_{T}^{2}\right) R_{4}\left(\boldsymbol{q}_{T}^{2}\right) .
\end{aligned}
$$



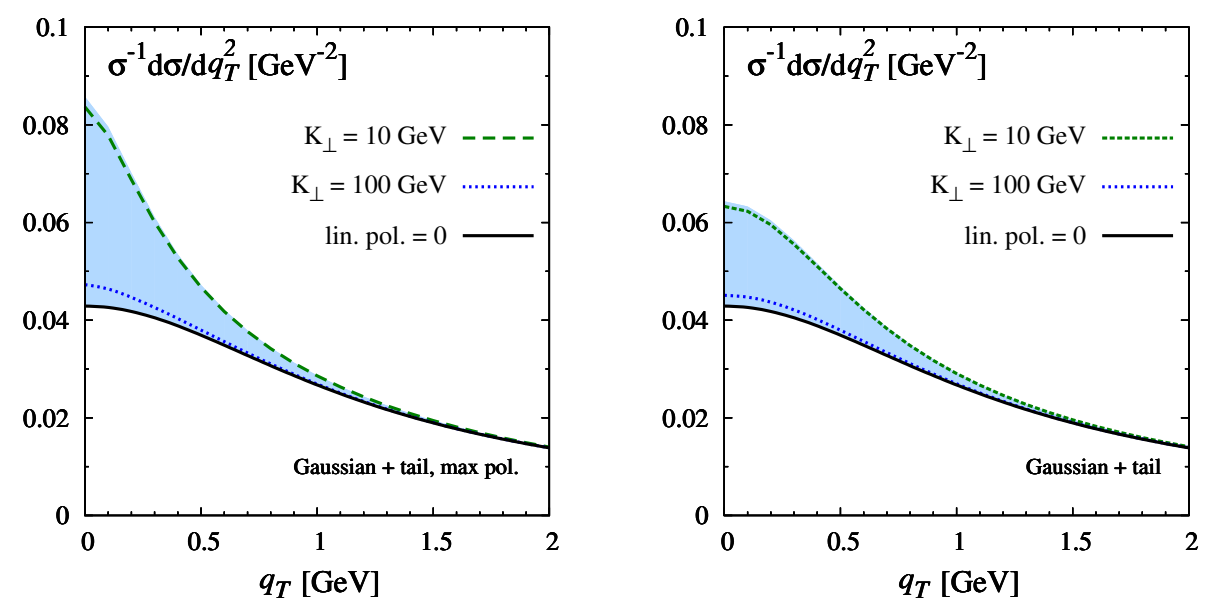

Figure 1: Transverse momentum distribution of the Higgs boson plus jet pair in the process $p p \rightarrow H$ jet $X$ for two different choices of $K_{\perp}, K_{\perp}=10$ and $100 \mathrm{GeV}$, with $q_{T \max }=M_{H} / 2$ and $y_{H}=y_{\mathrm{j}}$. The solid line indicates the distribution in absence of linear polarization. The shaded blue area represents the range of the spectrum as $K_{\perp}$ varies from zero to infinity.

\section{Numerical results}

In this section we provide numerical estimates for the observables in Eqs. (2.14)-(2.16), in the specific configuration in which the Higgs boson and the jet have the same rapidities $\left(y_{H}=y_{\mathrm{j}}\right)$. To this aim, we assume that the so far unknown unpolarized gluon TMD has the following form [4],

$$
f_{1}^{g}\left(x, \boldsymbol{p}_{T}^{2}\right)=f_{1}^{g}(x) \frac{R^{2}}{2 \pi} \frac{1}{1+\boldsymbol{p}_{T}^{2} R^{2}},
$$

where $R=2 \mathrm{GeV}^{-1}$ and $f_{1}^{g}(x)$ is the common gluon distribution, integrated over the transverse momentum. In order to show the maximal effects of gluon polarization, we take $h_{1}^{\perp g}\left(x, \boldsymbol{p}_{T}^{2}\right)$ to be positive and saturating its positivity bound in Eq. (2.5),

$$
h_{1}^{\perp g}\left(x, \boldsymbol{p}_{T}^{2}\right)=\frac{2 M_{p}^{2}}{\boldsymbol{p}_{T}^{2}} f_{1}^{g}\left(x, \boldsymbol{p}_{T}^{2}\right) .
$$

Alternatively, in analogy to Eq. (3.1), we consider the following model as well, for which the bound is saturated only in the limit $p_{T} \rightarrow \infty$ [4]:

$$
h_{1}^{\perp g}\left(x, \boldsymbol{p}_{T}^{2}\right)=c f_{1}^{g}(x) \frac{M_{p}^{2} R_{h}^{4}}{2 \pi} \frac{1}{\left(1+\boldsymbol{p}_{T}^{2} R_{h}^{2}\right)^{2}},
$$

with $c= \pm 2$ and $R_{h}=3 R / 2$. We note that there are no indications available on the actual shape of $h_{1}^{\perp g}$, therefore our models are only intended to illustrate what kind of features can arise qualitatively from linearly polarized gluons and to give an indication of the maximal effects that one might expect.

Our predictions for the transverse momentum distribution defined in Eq. (2.14) are presented in Fig. 1 for two different values of $K_{\perp} \equiv\left|\boldsymbol{K}_{\perp}\right|: 10$ and $100 \mathrm{GeV}$. In the left panel of all the figures, $h_{1}^{\perp g}$ is assumed to be maximal, see Eq. (3.2), while in the right one it is given by Eq. (3.3). 

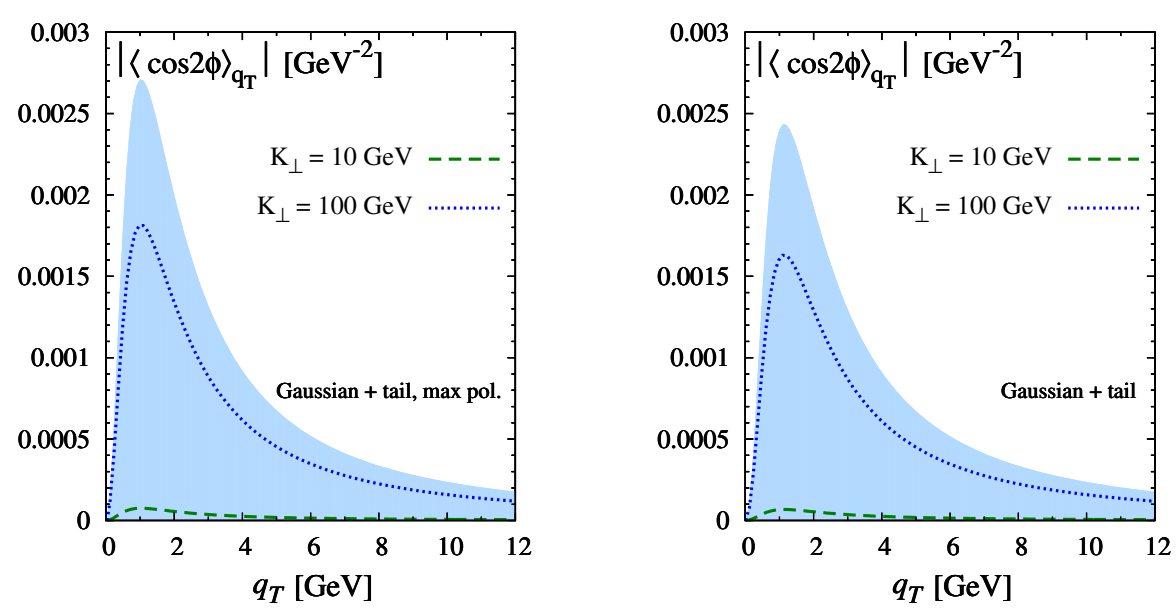

Figure 2: Absolute value of the $\langle\cos 2 \phi\rangle_{q_{T}}$ asymmetries the process $p p \rightarrow H$ jet $X$ for two different choices of $K_{\perp}, K_{\perp}=10$ and $100 \mathrm{GeV}$, with $q_{T \max }=M_{H} / 2$ and $y_{H}=y_{\mathrm{j}}$. The shaded blue area represents the range of the asymmetries as $K_{\perp}$ varies from zero to infinity.

Among all the observables discussed here, $\langle\cos 2 \phi\rangle_{q_{T}}$ is the only one which is sensitive to the sign of $h_{1}^{\perp g}$, and it is expected to be negative if $h_{1}^{\perp g}>0$. Its absolute value as a function of $q_{T}$ is shown in Fig. 2. Furthermore, we find that $|\langle\cos 2 \phi\rangle| \approx 12 \%$ if $K_{\perp}=100 \mathrm{GeV}$, while it is about $0.5 \%$ if $K_{\perp}=10 \mathrm{GeV}$. Analogously, our estimates for $\langle\cos 4 \phi\rangle_{q_{T}}$ are presented in Fig. 3, with $\langle\cos 4 \phi\rangle \approx 0.2 \%$ at $K_{\perp}=100 \mathrm{GeV}$ and completely negligible at $K_{\perp}=10 \mathrm{GeV}$. Very similar values are obtained if one considers a Gaussian model for $f_{1}^{g}$ and maximal gluon polarization [6].

\section{Conclusions}

We have presented several observables for the process $p p \rightarrow H$ jet $X$, which are sensitive to the polarized gluon TMD $h_{1}^{\perp g}$. We note that the proposed measurements are challenging, since
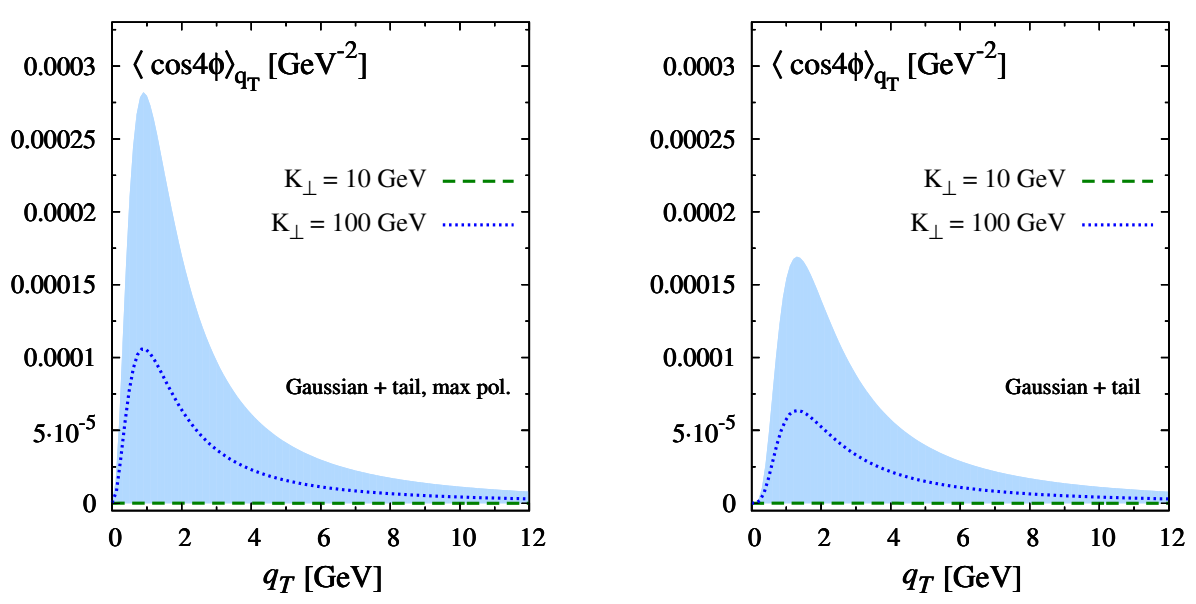

Figure 3: Same as in Figure 2, but for the $\langle\cos 4 \phi\rangle_{q_{T}}$ asymmetries. 
one would need several bins in $q_{T}$ in the kinematic region up to about $10 \mathrm{GeV}$. Therefore a high resolution of the transverse momentum of both the Higgs boson and the jet is required, in addition to the knowledge of how well the jet axis coincides with the direction of the fragmenting parton.

To conclude, Higgs production at the LHC, both inclusive and in association with a jet, complemented by the study of processes likes $p p \rightarrow \eta_{c, b} X, p p \rightarrow \chi_{0 c, b} X$ [9] and $p p \rightarrow J / \psi \gamma X$ [7], can be used to access $h_{1}^{\perp g}$ and to analyze its process and scale dependences. Additional information might be gathered by looking at the electroproduction of heavy quark pairs and dijets $[10,11]$ that could be measured at future EIC or LHeC experiments.

\section{Acknowledgments}

We acknowledge support by the Fonds Wetenschappelijk Onderzoek - Vlaanderen (FWO) through a Pegasus Marie Curie Fellowship.

\section{References}

[1] P. J. Mulders and J. Rodrigues, Transverse momentum dependence in gluon distribution and fragmentation functions, Phys. Rev. D 63 (2001) 094021 [arXiv : hep-ph / 000934 3].

[2] D. Boer, W. J. den Dunnen, C. Pisano and M. Schlegel, Determining the Higgs spin and parity in the diphoton decay channel, Phys. Rev. Lett. 111 (2013) 032002 [arXiv:1304.2654 [hep-ph] ].

[3] D. Boer, W. J. den Dunnen, C. Pisano, M. Schlegel and W. Vogelsang, Linearly Polarized Gluons and the Higgs Transverse Momentum Distribution, Phys. Rev. Lett. 108 (2012) 032002 [arXiv:1109.1444 [hep-ph]].

[4] D. Boer and W. J. den Dunnen, TMD evolution and the Higgs transverse momentum distribution, Nucl. Phys. B 886 (2014) 421 [arXiv: 1404.6753 [hep-ph] ].

[5] M. G. Echevarria, T. Kasemets, P. J. Mulders and C. Pisano, QCD evolution of (un)polarized gluon TMDPDFs and the Higgs $q_{T}$-distribution, to appear in JHEP (2015) [arXiv: 1502.05354 [hep-ph]].

[6] D. Boer and C. Pisano, Impact of gluon polarization on Higgs boson plus jet production at the LHC, Phys. Rev. D 91 (2015) 074024 [arXiv:1412.5556 [hep-ph] ].

[7] W. J. den Dunnen, J. P. Lansberg, C. Pisano and M. Schlegel, Accessing the Transverse Dynamics and Polarization of Gluons inside the Proton at the LHC, Phys. Rev. Lett. 112 (2014) 212001 [arXiv:1401.7611 [hep-ph]].

[8] T.C. Rogers and P.J. Mulders, No Generalized TMD-Factorization in Hadro-Production of High Transverse Momentum Hadrons, Phys. Rev. D 81 (2010) 094006 [arXiv: 1001.2977 [hep-ph]].

[9] D. Boer and C. Pisano, Polarized gluon studies with charmonium and bottomonium at LHCb and AFTER, Phys. Rev. D 86 (2012) 094007 [arXiv:1208.3642 [hep-ph]].

[10] D. Boer, S. J. Brodsky, P. J. Mulders and C. Pisano, Direct Probes of Linearly Polarized Gluons inside Unpolarized Hadrons, Phys. Rev. Lett. 106 (2011) 132001 [arXiv:1011.4225 [hep-ph]].

[11] C. Pisano, D. Boer, S. J. Brodsky, M. G. A. Buffing and P. J. Mulders, Linear polarization of gluons and photons in unpolarized collider experiments, JHEP 1310 (2013) 024 [arXiv: 1307.3417 [hep-ph] ]. 